Rhétorique et réalité : les collectivités françaises et leurs voisins du Pacifique Sud

Rhetoric and Reality: the French Territories and their Neighbours

\title{
Denise Fisher
}

\section{(2) OpenEdition Journals}

Édition électronique

URL : http://journals.openedition.org/jso/7239

DOI : $10.4000 /$ jso.7239

ISSN : $1760-7256$

Éditeur

Société des océanistes

Édition imprimée

Date de publication : 15 juin 2015

Pagination : $39-48$

ISBN : 9782854301250

ISSN : 0300-953x

Référence électronique

Denise Fisher, «Rhétorique et réalité : les collectivités françaises et leurs voisins du Pacifique Sud », Journal de la Société des Océanistes [En ligne], 140 I janvier-juin 2015, mis en ligne le 05 juillet 2015, consulté le 20 avril 2019. URL : http://journals.openedition.org/jso/7239 ; DOI : 10.4000/jso.7239 


\section{Rhétorique et réalité : les collectivités françaises et leurs voisins du Pacifique Sud}

par

Denise FISHER ${ }^{*}$

\section{RÉSUMÉ}

Les dirigeants français ont engagé une politique d'insertion des collectivités ultramarines dans leurs environnements régionaux. Dans le Pacifique Sud, la France a fait des progrès solides en assurant la participation officielle de ses collectivités dans la gamme d'organisations régionales, dans la coopération régionale de défense, et en soutenant les collaborations plus officieuses, telles que culturelles et sportives. La France a également stimulé l'intérêt de l'Union européenne pour la région. Mais il reste encore beaucoup à faire. Plusieurs aspects de cette politique freinent plutôt qu'ils ne renforcent l'insertion des collectivités dans leur région. En même temps la France n'est pas encore au bout des processus qu'elle a mis en place pour répondre aux demandes des indépendantistes et des autonomistes en Nouvelle-Calédonie et en Polynésie française. L'insertion et l'acceptation régionales de ses collectivités exigent la résolution réussie de ces questions.

MoTS-CLÉS : collectivités françaises, insertion régionale, Nouvelle-Calédonie, Polynésie française, CPS

\section{Politique d'insertion régionale pour l'Outre-mer}

Les gouvernements successifs de la République ont affirmé une politique d'insertion des collectivités d'Outre-mer dans leurs environnements régionaux respectifs. Le président Sarkozy avait défini cette politique quand il avait proposé une série de réformes comme réponse aux manifestations contre la vie chère en 2009, qui avaient commencé en Guadeloupe mais s'étaient vite étendues à la Martinique, la Guyane et La Réunion.

\section{ABSTRACT}

French leaders have declared a policy of integration of their overseas territories into their respective regions. In the Pacific, France has made solid progress in encouraging the official participation of its entities in the range of regional organisations, regional defence cooperation, and informally through sporting and cultural events. France has also led European Union engagement in the region. But a lot more needs to be done. Many aspects of France's integration policy work more to separate their collectivities from the region rather than to strengthen their regional integration. At the same time, France has not yet finalised the processes put in place to respond to those seeking autonomy and independence in New Caledonia and French Polynesia. Full regional integration and acceptance of its collectivities requires successful resolution of these issues.

KeYwords: French territories, regional cooperation, New Caledonia, French Polynesia, sPC

François Hollande avait déclaré à son tour, le 19 novembre 2012, que l'insertion des collectivités dans leur environnement international était dans l'intérêt de la France (Hollande, 2012). Il voulait que les collectivités d'Outre-mer représentent la France dans les organisations régionales. Son ministre des Outre-mer, Victorin Lurel, avait parlé de l'insertion régionale en soulignant, lors du conseil des ministres du 6 juin 2013, l'importance stratégique des Outre-mer pour la France. Il avait évoqué la collaboration de l'Union européenne (UE) et

* Chercheur invité, Centre des études européennes, Australian National University (ANU) et ancien consul général de l'Australie à Nouméa (2001-2004), denisemfisher@gmail.com 
de ses ртом (pays et territoires d'Outre-mer), en soulignant que les Outre-mer agiraient « comme acteurs de la politique d'aide et de développement de la France dans les pays environnants " surtout dans les secteurs énergétiques, dans "l'économie du savoir », et dans la santé (Lurel, 2013).

\section{Actions dans la région du Pacifique Sud: la France}

Il y a longtemps que la France a soutenu une politique d'insertion régionale pour ses trois territoires du Pacifique Sud : la Nouvelle-Calédonie, la Polynésie française et Wallis-et-Futuna. Pour faciliter la lecture de ce papier, l'auteur utilisera le terme de "collectivités françaises du Pacifique Sud " pour désigner ces trois collectivités, quoique selon l'article 74 de la constitution (après un changement en 2003), la Polynésie française et Wallis-et-Futuna soient des collectivités territoriales d'outre-mer alors que la Nouvelle-Calédonie est une collectivité spécifique relevant du titre XIII de la constitution. Afin de rattraper les pertes (quant à son image et sa réputation internationale) après les controverses autour de ses politiques d'expérimentation nucléaire et de résistance à la décolonisation, durant les années 1980, la France a conçu toute une série de mesures pour démontrer aux leaders océaniens qu'elle avait changé, qu'elle voulait non seulement régler ces deux questions si épineuses, mais qu'elle voulait aussi des liens approfondis avec les organisations, gouvernements et leaders politiques du Pacifique Sud. Les deux mesures les plus importantes furent la cessation définitive des essais nucléaires en Polynésie française ${ }^{1}$ et la négociation réussie des accords de Matignon-Oudinot (1988) puis de Nouméa (1998), ces derniers étant inscrits dans la constitution de la République (articles 76 et 77). L'accord de Nouméa représente un processus de reconnaissance de l'identité et des aspirations, non seulement des " nouveaux arrivés " en Nouvelle-Calédonie (c'est-à-dire les Européens - à savoir les anciens colons et fermiers, dits Caldoches et les métropolitains - mais aussi des autres populations ultramarines françaises ainsi que des immigrants d'Océanie et d'ailleurs), mais surtout des peuples autochtones.

Depuis 1989, la France est partenaire au dialogue du forum des îles du Pacifique, l'organisation principale de la région Elle a renforcé sa participation à l'organisation technique qu'est la CPS (secrétariat de la communauté du Pacifique) dont d'ailleurs Nouméa abrite le siège, et à ses organisations subrégionales (les organisations du CROP ou Council of Regional Organisations of the Pacific, conseil des organisations régionales) qui forment le cœur de la coopération technique, pratique et quotidienne de la région. La France conduit des exercices militaires, non seulement avec les grands pays du Pacifique, l'Australie et la Nouvelle-Zélande, mais aussi avec les États insulaires voisins (Papouasie Nouvelle-Guinée [PNG], Tonga, Vanuatu). Avec l'Australie et la Nouvelle-Zélande, la France a formé un accord FRANZ, arrangement utilisant leurs atouts de défense et d'aide d'urgence pour aider les États insulaires à la surveillance des zones maritimes et de pêche et pour répondre rapidement aux cas d'urgence tels que les catastrophes climatiques. Avec ces pays et les États-Unis, elle participe aux discussions quadrilatérales sur la sécurité régionale. Elle a œuvré pour une participation renforcée de l'Union européenne dans la région, non seulement grâce à l'aide au développement mais aussi en permettant aux économies insulaires de pénétrer le marché attractif de l'Europe.

La France contribue à de nombreux fonds du Pacifique Sud, par le biais de ses contributions à la CPS, son aide de coopération bilatérale et ses dotations au Fonds de développement européen (FED), auquel la France contribue à hauteur de $20 \%$ de sa totalité. Dans les années 2000, le montant total équivalait à environ 100 millions d'euros par an. Et depuis 2003, la France organise tous les trois ans environ des sommets océaniens avec les États indépendants de la région. Ces sommets s'inscrivent dans une politique d'encouragement à l'intégration des entités françaises du Pacifique dans leur environnement régional.

\section{Actions dans la région: ses collectivités océaniennes}

La France a ouvert la voie, après des décennies, et même une centaine d'années d'isolement, à la participation de ses trois collectivités à la vie de la région. Et ce sens, l'isolement était bien réel : par exemple, ce n'est qu'en 1981 que, pour la première fois, la France a permis aux hauts-commissaires et au préfet des collectivités, d'entretenir des relations avec les ambassadeurs français de la région, ce qui était interdit avant cette date (Sénat, 2013a : 20).

L'accord de Nouméa de 1998 et la loi organique pour la Polynésie française de 2004 ont accordé à ces deux collectivités leurs propres compétences extraterritoriales. Ces pouvoirs sont éloquents et représentent en effet un partage de souveraineté. Ils incluent le pouvoir de représentation en leur propre titre auprès des pays du Pacifique, des organisations régionales et même de l'uE et des Nations unies. Ces collectivités ont leurs compétences particulières pour le commerce extérieur, les services maritimes et aériens. Elles peuvent négocier avec les pays du Pacifique $\mathrm{Sud}^{2}$. Mais il

1. En 1996, avec la signature par la France du traité d'interdiction complète des essais nucléaires (TICE).

2. L'accord de Nouméa de 1998 spécifie les compétences du gouvernement local de la Nouvelle-Calédonie pour quelques éléments du commerce extérieur, pour les services maritimes et aériens (article 3.1.1), sa propre représentation 
va sans dire que ces partages de souveraineté s'inscrivent dans les pouvoirs souverains continus et supérieurs de l'État. L'accord de Nouméa spécifie ces pouvoirs régaliens de l'État (défense, affaires étrangères, justice, ordre public, monnaie) que la France détient au moins jusqu'à un processus de référendum prévu entre 2014 et 2018.

La France a obtenu un statut particulier pour ses trois collectivités au sein du forum des îles du Pacifique, en première instance comme observateurs (Nouvelle-Calédonie 1999, Polynésie française 2004, Wallis-et-Futuna 2006) et ensuite, dès 2006, comme "membres associés " pour les deux premières.

La France a soutenu la participation à part entière (c'est-à-dire en leur propre nom) de ses collectivités à la CPS, et dans un grand nombre d'organisations du CROP, organisations importantes, car engagées dans le travail pratique et technique au quotidien sur les sujets fondamentaux pour la région, tels que l'environnement, la géoscience, la pếche, le tourisme (voir tableau 1). En juillet 2013, la France a soutenu la participation des deux universités, de Nouvelle-Calédonie et de Polynésie française, à un réseau de coopération universitaire du Paci- fique Sud. Les collectivités participent aux activités culturelles et sportives de la région, surtout aux jeux du Pacifique, auxquels elles contribuent aussi en tant que pays organisateur en alternance avec les autres pays océaniens.

Le Fonds Pacifique a été établi en 1987 pour améliorer l'image de la France dans la région par le biais de l'aide au développement sous l'égide du premier secrétaire d'État pour le Pacifique Sud, Gaston Flosse. Progressivement, le fonds s'est transformé jusqu'à devenir aujourd'hui un fonds dédié spécifiquement à l'insertion régionale des collectivités françaises. Il est de plus en plus géré par elles, bien que sous la tutelle des hautscommissaires français et soutenu par le secrétariat permanent pour le Pacifique basé à Paris.

À leur crédit, les deux grandes collectivités ont vite lancé des programmes, quoique de petites tailles et limités, d'aide à la coopération avec leurs voisins immédiats. La Polynésie française a commencé en 2002 par l'aide d'urgence à Tonga après un cyclone. La Nouvelle-Calédonie a conclu un accord commercial avec l'Australie en 2002 et une convention de coopération avec le Vanuatu la même année. Et la Nouvelle-Calé-

TABleau 1. - Participation des collectivités françaises - organisations régionales du Pacifique Sud (source :

Fisher, 2013 : 208, utilisant les données statistiques de l'ISEE TEC 2008 : 13)

\begin{tabular}{|l|c|c|c|}
\hline Organisation & $\begin{array}{c}\text { Nouvelle- } \\
\text { Calédonie }\end{array}$ & $\begin{array}{c}\text { Polynésie } \\
\text { française }\end{array}$ & $\begin{array}{c}\text { Wallis-et- } \\
\text { Futuna }\end{array}$ \\
\hline $\begin{array}{l}\text { Secrétariat de la communauté du Pacifique (Secretariat for the } \\
\text { Pacific Community) }\end{array}$ & $\mathrm{M}$ & $\mathrm{M}$ & $\mathrm{M}$ \\
\hline Forum des îles du Pacifique (Pacific Islands Forum) & $\mathrm{AM}$ & $\mathrm{AM}$ & $\mathrm{O}$ \\
\hline $\begin{array}{l}\text { Commission du Pacifique Sud pour les géosciences appliquées } \\
\text { (South Pacific Applied Geoscience Commission) }\end{array}$ & $\mathrm{AM}$ & $\mathrm{AM}$ & \\
\hline $\begin{array}{l}\text { Agence des Pêches } \\
\text { (Forum Fisheries Agency) }\end{array}$ & $\mathrm{O}$ & \\
\hline $\begin{array}{l}\text { Conseil coopération économique Pacifique (Pacific Economic } \\
\text { Cooperation Council) }\end{array}$ & $\mathrm{AM}^{\mathrm{f}}$ & $\mathrm{AM}^{\mathrm{f}}$ & $\mathrm{AM}^{\mathrm{f}}$ \\
\hline $\begin{array}{l}\text { Organisation du Tourisme du Pacifique Sud (South Pacific } \\
\text { Tourism Organization) }\end{array}$ & $\mathrm{M}$ & $\mathrm{M}$ & \\
\hline $\begin{array}{l}\text { Programme de développement des îles du Pacifique (Pacific } \\
\text { Islands Development Program) }\end{array}$ & $\mathrm{M}$ & $\mathrm{M}$ & $\mathrm{M}$ \\
\hline $\begin{array}{l}\text { Programme régional Océanie de l'environnement (South Pacific } \\
\text { Regional Environment Program) }\end{array}$ & $\mathrm{M}$ & $\mathrm{M}$ & $\mathrm{M}$ \\
\hline $\begin{array}{l}\text { Association des producteurs d'électricité du Pacifique (Pacific } \\
\text { Power Association) }\end{array}$ & $\mathrm{M}$ & $\mathrm{M}$ & $\mathrm{M}$ \\
\hline $\begin{array}{l}\text { Organisation douanière du Pacifique (Oceanic Customs } \\
\text { Organisation) }\end{array}$ & $\mathrm{M}$ & $\mathrm{M}$ & \\
\hline
\end{tabular}

M : Membre

AM : Membre associé

$\mathrm{O}$ : Observer
$\mathrm{AM}^{\mathrm{f}}:$ Membre associé avec la France
* Organisation membre du conseil des organisations régionales (Council of Regional Organisations of the Pacific)

auprès des pays du Pacifique Sud, et auprès de quelques organisations régionales, de l'UE, et des Nations unies, ainsi que le pouvoir de négocier des accords avec les pays de la région (article 3.2.1). La loi organique pour la Polynésie française de 2004 octroie également au gouvernement local de la Polynésie française le pouvoir de représentation auprès des autres pays (quoique le conseil constitutionnel ait qualifié ce pouvoir comme moindre de la pleine représentation diplomatique). Cette loi donne au président du territoire le pouvoir de négocier des accords administratifs avec tout État ou territoire du Pacifique pour avancer son développement économique ou social et de signer les accords dans les secteurs de la compétence de la Polynésie française (articles 15-17). La Polynésie française peut devenir membre, associé, ou observateur auprès des organisations internationales et le président peut s'associer aux organisations du Pacifique Sud dans les secteurs de la compétence de la Polynésie française (article 42). 
donie, en particulier, se montre de plus en plus ouverte pour accueillir des réunions internationales à Nouméa. En 2010, elle fut l'hôte du festival des arts mélanésiens et, en 2011, celle des jeux du Pacifique (elle a même offert alors une zone libre de visas de court séjour aux participants régionaux). Encouragé par l'État français, elle a aussi accueilli à plusieurs reprises les représentants du groupe du Fer de Lance mélanésien (GFLm, Melanesian Spearhead Group), dont le groupe politique FLNKS est membre, et elle a même exprimé le désir d'y appartenir à part entière.

Dès lors, on pourrait dire qu'apparemment, la République a investi de fortes ressources diplomatiques et politiques ces dernières années pour démontrer l'importance qu'elle attache à ce que ses collectivités appartiennent et participent aux organisations et à la vie régionale. La France a réalisé un grand effort, non seulement au sein de ses collectivités, mais plus largement pour nouer des liens avec les pays et les organisations de la région. Mais quelques-unes de ses actions pourraient laisser croire, au moins aux petits États insulaires de la région, que la France et les collectivités, et même l'Union européenne, n'envisagent pas assez sérieusement la mise en œuvre de leurs intentions déclarées, d'insérer les collectivités françaises d'une manière réelle et pratique, dans la vie de la région.

\section{Aide à la coopération : chiffres modestes et obscurs}

En premier lieu, les traits qui caractérisent l'aide à la coopération de la France et de l'ue reflètent parfaitement les difficultés qui compliquent l'image de la France dans la région.
Bien sûr, cette aide est la bienvenue et très bien reçue par les pays petits, insulaires et isolés. Mais il y a un manque de transparence sur les chiffres et sur l'effet de cette aide à la coopération, ce qui fait qu'il est impossible de savoir exactement combien la France et l'Ue versent aux pays du Pacifique. Même notre recherche intensive n'a pas permis de produire davantage qu'un petit tableau incomplet et ponctué de notes de clarification et de qualification (tableau 2). Les meilleurs chiffres disponibles montraient que :

- La France verse à peu près 103 millions d'euros par an au Pacifique Sud.

- L'ue verse à peu près 90 millions d'euros par an, dont $20 \%$ représentent la contribution de la France.

Non seulement on ne peut pas facilement identifier les montants des activités diverses, mais souvent ces allocations elles-mêmes ne reflètent pas la réalité. Trop souvent elles ne sont pas entièrement dépensées, en raison des systèmes éloignés et lourds des formulaires, des exigences bureaucratiques et administratives qui dépassent la capacité des petits États insulaires. En outre, il y a des ambiguïtés, car certains chiffres français comprennent la contribution de la France, ou une partie de sa contribution, par voie de l'ue, et d'autres chiffres ne l'incluent pas, ce qui n'est pas toujours clair (même dans les chiffres cités ci-dessus).

Par contre, les chiffres du soutien de la République à ses propres collectivités du Pacifique Sud sont tout à fait disponibles. Toute la région peut facilement établir que la France attribue plus d'un milliard d'euros par an à chacune de ses grandes collectivités, la Nouvelle-Calédonie et la Polynésie française (2,5 milliards d'euros pour 2013 ; Laurin, 2013 : 28).

Ainsi, l'aide de l'État à la région ne représente que $3,4 \%$ de son soutien à ses collectivités du

TABleau 2. - Chiffres indicatifs sur l'aide de coopération de la France à la région du Pacifique Sud (en millions d'euros et en millions de dollars australiens : \$A) (source : Fisher, 2013 : 198)

\begin{tabular}{|c|c|c|c|}
\hline & $2006^{*}$ & $2007^{\#}$ & $2008^{\#}$ \\
\hline Aide à la région ${ }^{*}$ & $278(\$ A 40)$ & $98(140)$ & $103(146)$ \\
\hline Dont bilatérale $^{*}$ & $15,0(\$ \mathrm{~A} 21)$ & & \\
\hline Dont celle par voie de l'UE (environ $20 \%$ FDE) & $12,8(\$ \mathrm{~A} 19)$ & & \\
\hline \multicolumn{4}{|l|}{ Quelques programmes soutenus (incomplet): } \\
\hline Fonds Pacifique Sud & \multicolumn{3}{|c|}{$2,4(\$ A 3,4)$ moyenne par an 2007-2009 } \\
\hline $\mathrm{CPS}^{+}$contribution bilatérale & \multirow{2}{*}{\multicolumn{3}{|c|}{ 3,0 (\$A4,2) moyenne par an 1999-2009 }} \\
\hline Et contribution française par voie de l'UE & \multirow{2}{*}{\multicolumn{3}{|c|}{$\frac{1,0(\$ \mathrm{~A} 1,4) \text { moyenne par an 2002-2007 }}{2,0(\$ \mathrm{~A} 2,8) \text { moyenne par an 2004-9 }}$}} \\
\hline Initiative Récifs 2004-2009 & & & \\
\hline $\begin{array}{l}\text { Activités par le biais de FRANZ }{ }^{++} \\
\text {(aide d'urgence, soutien logistique) }\end{array}$ & \multicolumn{3}{|c|}{$1,0(1,4)$} \\
\hline
\end{tabular}

* Ministère des Affaires étrangères et du Développement international (www.diplomatie.gouv.fr/fr/pays-zones-geo_833/ oceanie_14692/index.html, consulté le 14 mai 2009)

\# Ministère des Affaires étrangères et du Développement international, site web consulté le 26 février 2010, peut inclure les allocations par le biais des programmes de l'uE

${ }^{+}$CPS Annual Reports and Financial Statements, France and EU Support to SPC 1993 to 2009.

${ }^{++}$Estimations du FANC 
Pacifique Sud. Cela ne constitue que $2 \%$ de l'aide à la coopération globale de la France, très peu quand on pense que celle-ci est présente dans cette région en tant que puissance souveraine. Paradoxalement, la France attribue $53 \%$ de son aide en Afrique, où elle n'a pas de présence souveraine (ministère français des Affaires étrangères, 2010). Et par rapport aux sommes attribuées, annuellement, par l'Australie $(1,09$ milliard de \$A; ou 0,774 milliard d'euros) et même par la Nouvelle-Zélande (205,5 millions de $\$$ A ou 145 millions d'euros) la contribution de la France n'est pas énorme (Australian Minister for Foreign Affairs, 2009, NZ AID, 2010)3.

De plus, la France a mis en ouvre depuis des années une politique pour assurer que les collectivités elles-mêmes soient les bénéficiaires de l'aide française versée dans la région. Par exemple, en 1996, quand il a ratifié une convention régionale sur l'environnement, le sénat a noté que :

«La politique de la France a évolué jusqu'au point où sa participation est conditionnelle à l'engagement de ses collectivités [...]. L'aide française au développement du Pacifique Sud doit aussi bénéficier aux territoires français. [...] La France a toujours conditionné sa contribution aux programmes du forum du Pacifique Sud à la participation de nos territoires aux projets de développement ainsi financés. Parmi les projets du forum auxquels a contribué le "Fonds Pacifique", relevons notamment la promotion du commerce avec les том français de la région. » (Sénat, 1996)

Le manque de transparence des chiffres et les ambiguïtés qui les entourent sont dommageables pour l'image de la France et de ses collectivités. $\mathrm{Au}$ lieu de présenter clairement et positivement les sommes qui, quoique modestes, peuvent tout de même faire une vraie différence dans la vie des gens des petites îles, l'absence de chiffres clairs peut laisser croire à une intention de voiler ou de déguiser la réalité. Et cela n’aide pas à renforcer la confiance envers les intentions et la participation de la France et de ses collectivités dans la région.

\section{Le commerce}

Concernant le commerce, l'État parle d'une participation des collectivités dans leur région, et même de l'intention de faire baisser le coût de la vie accablant dans ces dites collectivités. Mais il continue à refuser fermement à ses collectivités l'achat local, auprès des États voisins, répondant à leurs besoins, et à un prix raisonnable. Les taxes imposées par les collectivités à l'entrée des produits des pays voisins restent très élevées. De plus, l'État continue à encourager activement l'importation presque exclusive par les collecti- vités de produits venus de France et d'Europe, qui viennent donc de très loin, avec tous les frais de frets et les coûts élevés de production qui y sont afférents et contribuent ainsi à maintenir des prix élevés.

La France a aussi mené une politique de changement du traitement des ртом, les Pays et territoires Outre-mer de l'Union européenne, et des pays insulaires ACP (Afrique-Caraïbes-Pacifique) depuis 2009, changements qui servent à distinguer ses collectivités des pays insulaires plutôt qu'à contribuer à l'intégration régionale. Ces changements aboutirent à la décision 2013/755/ UE du conseil de l'UE, le 25 novembre 2013, relative à l'association des pays et territoires d'outre mer à l'Union européenne (décision d'association outre mer). Cette décision approuvait de faire reposer le futur partenariat entre l'Union et les Ртом sur les trois piliers que sont le renforcement de la compétitivité, le développement de la capacité et la réduction de la vulnérabilité, l'action en faveur de la coopération et de l'intégration entre les PTOM et d'autres partenaires et régions voisins. Quoique, par cette décision, l'us ait adopté elle-même la politique d'insertion régionale pour les ртом menée par la France (voir par exemple Saramandis 2013: 49), la réalité et les effets de sa politique sont autres. Les PTOM, y compris les collectivités du Pacifique Sud, sont traités de plus en plus nettement comme des pays membres de l'ue. Ils bénéficient des programmes normaux de l'UE, car de moins en moins traités comme dépendants de l'aide à la coopération et de plus en plus comme partenaires dans les programmes de développement durable, l'accent étant mis sur la compétitivité (Commission, 2009). Ils sont maintenant décrits comme « les avant-postes stratégiques " non seulement de la France mais aussi de l'Europe. Ce processus est perçu dans la région comme «l'européanisation » des collectivités françaises du Pacifique Sud, plutôt que l'insertion de ces collectivités dans leur région (Muller et Blockmans, in Kochenov (ed.), 2011 ; Calvignac, 2013 : 55-57 ; Fisher, 2012b). Alors que les ртом sont traités de plus en plus comme une partie de la famille européenne, l'UE, avec la forte participation de la France, a changé l'accès préférentiel des petites îles des ACP au marché de l'Europe. Ainsi, les petits pays insulaires ont perdu leurs droits d'accès privilégié, qui vont être remplacés par un schéma "APE " (accords de partenariat économique). C'est une structure essentiellement européenne, imposée à la région sans considérer les structures et projets locaux, tels que le Plan océanien (Pacific Plan, architecture régionale pour la coopération), le PICTA (Pacific Island Countries Trade Agreement, instrument de libre échange régional et exclusif aux petits États insulaires) et le PaCER (Pacific

3. Les chiffres datent de 2009, même période que les chiffres les plus récents pour la France. 
Agreement on Closer Economic Relations, accord entre les petits États insulaires, l'Australie, et la Nouvelle-Zélande), avec lesquels les APE ne sont pas du tout compatibles. Ces trois accords/plans sont multilatéraux, et partent du principe que chaque participant agit dans l'intérêt régional, tandis que les APE sont des accords bilatéraux entre l'UE et chaque gouvernement insulaire.

Pourquoi s'étonner si, après des années de négociations et de discussions, l'échéance APE de 2008 n'a abouti à aucun accord conclu, et que même aujourd'hui, il n'en soit résulté que deux accords, avec les deux puissances économiques insulaires de la région, la Papouasie-Nouvelle-Guinée et Fidji ? Comment inclure les États plus petits qui, en ce moment, ne bénéficient que des tarifs préférentiels généralisés? Quant aux collectivités françaises, jusqu'à présent, seule la Nouvelle-Calédonie a parlé de " considérer » sa participation dans les projets PICTA et PACER, sans entreprendre aucun changement de ses propres politiques pour accommoder un accès plus ouvert aux États insulaires.

Quel est le résultat de cette politique? En 2013, les exportations de la Nouvelle-Calédonie, la plus grande des économies des collectivités françaises, se sont dirigées principalement vers l'Ue (dont plus de la moitié vers la France), puis le Japon, l'Australie, et la Corée du Sud. Ses importations sont venues, elles aussi, principalement de l'ue (dont les deux tiers de France), de Singapour, d'Australie, des États-Unis, et de la Nouvelle-Zélande. Son commerce avec les pays insulaires de la région a été si minime que les statistiques officielles n'en font même pas mention (ISEE site web, consulté le 15 janvier 2015). En 2012, l'Australie ne représentait que $10,9 \%$ du marché en Nouvelle-Calédonie (après la France et Singapour) (DFAT, 2015). Par contre, l'ue ne reçoit que $10 \%$ des exportations des pays ACP du Pacifique Sud, dont plus de $90 \%$ provenaient de PNG et de Fidji (Commission, 2006 : 24, chiffres les plus récents disponibles).

Avec tous ces changements, la réduction de l'accès préférentiel des États indépendants aux marchés de l'Union européenne, l'exclusion continue de l'accès aux marchés des territoires français dans leur région et le traitement croissant des collectivités françaises en tant que partie intégrale de la France et de l'Europe, les petits États insulaires de la région ont raison de croire que la stratégie politique de la France consiste de plus en plus à conserver ses collectivités en son sein plutôt que de les insérer dans leur région.

Les disparités de richesse entre les trois collectivités françaises et les petits États insulaires restent énormes, avec par exemple un PIB par habitant en Nouvelle-Calédonie beaucoup plus élevé que celui des petites îles (et dans une certaine mesure, plus important même que celui de la NouvelleZélande ; CPS site web). Dans ces circonstances, ce serait aux collectivités françaises d'accorder les plus grandes concessions aux petits États pour contribuer réellement au développement de l'architecture commerciale de la région. C'est un geste essentiel pour la France et ses collectivités, si elles veulent leur acceptation par la région.

\section{Capacités des instances locales}

Quant à la participation des collectivités ellesmêmes dans les organisations régionales, il reste beaucoup à faire pour qu'elles puissent y participer de leur propre volonté. Certes, elles sont membres soi-disant « à part entière " de la plupart des organisations régionales. Mais que vaut ce droit si les représentants des collectivités elles-mêmes ne sont pas présents, aux réunions grandes et petites, pour décider de leurs positions à elles, sans avoir un conseil français à la tête de la délégation ou en base arrière, à Nouméa ou Papeete? Le Service de la coopération régionale et des relations extérieures du gouvernement de Nouvelle-Calédonie existe, mais comme l'explique le site web du gouvernement de la Nouvelle-Calédonie, il développe ces relations régionales "au côté de la France ", et ses positions sont finalement celles de la France.

Les collectivités se sont servies de leurs pouvoirs extérieurs avec plus ou moins d'enthousiasme mais toujours avec l'État en arrière plan. On pourrait dire que Gaston Flosse, en tant que Président de la Polynésie française, a même outrepassé ses compétences quand il a ouvert des bureaux de représentation de son gouvernement en Chine en mai 2004, et auprès de l'UE. Le conseil constitutionnel a vite jugé que ces représentations ne correspondaient pas à de pleines représentations diplomatiques :

«Cette faculté [compétence dotée par l'article 15 de la loi organique], qui n'appartenait pas jusqu'à présent à la Polynésie française, ne saurait conférer à ces représentations un caractère diplomatique. » (Conseil Constitutionnel, 2004)

Ni la Polynésie française ni la Nouvelle-Calédonie n'ont utilisé le pouvoir de négocier elles-mêmes avec l'UE, et elles ne participent aux réunions UE/PTOM qu'aux côtés de la France. En outre, la France n'a encouragé ni l'une ni l'autre à établir un bureau de représentation auprès des Nations unies.

Au niveau pratique, les notions innovatrices comme la Convention de Coopération avec le Vanuatu n'ont pas vraiment de substance si elles ne sont pas basées sur un soutien quotidien, grâce à un secrétariat bien équipé pour mettre en œuvre les promesses des accords, et une représentation sur place (voir Mrgudovic, 2010 : 209).

La France devrait avoir un peu plus confiance dans les citoyens calédoniens et polynésiens autochtones, ainsi qu'en ceux qui sont venus de l'Hexagone. Elle devrait les valoriser afin de montrer leur propre statut et leurs propres in- 
térêts. Ne pas le faire risquerait d'entraîner des résultats contre-productifs pour la France et les collectivités. Les gouvernements des États insulaires craignent d'accepter la pleine participation des collectivités (par exemple dans l'organisation prééminente, le FIP), tant que leurs statuts futurs n'auront été clarifiés, car ils craignent que les collectivités ne finissent par ne représenter que la position de la France. Parce que celle-ci a ses propres liens avec toutes les organisations régionales du Pacifique Sud (membre plein de la CPS, partenaire au dialogue du FIP), les leaders des îles indépendantes ne veulent pas que cette puissance européenne ait deux, trois ou quatre voix (par le biais des trois collectivités) dans ces organisations, ou dans les organisations du " CROP ». Il faut souligner que nous parlons ici des positions des gouvernements insulaires du FIP : depuis 2010 le gouvernement australien soutient la pleine participation des collectivités françaises du Pacifique Sud au sein du PIF (DFAT, 2010).

Et, chez eux, à Nouméa, Papeete et Mata Utu, il est à regretter qu'il n'existe pas de secrétariats permanents, même petits, mais qui soient vraiment indépendants de l'État et constitués d'habitants locaux y compris les autochtones, grâce auxquels les leaders locaux pourraient développer leur propre politique, leurs propres archives, leur propre participation pratique de jour en jour à toute la gamme des activités régionales.

Pour la Nouvelle-Calédonie, il serait même plus important d'établir un bureau "tout-en-un", car les contacts avec les niveaux de bureaucratie, pour les gouvernements externes, n'y sont pas du tout facilités. Avec des compétences variées définies par l'accord de Nouméa, on trouve que pour envisager un sujet particulier, il faut s'adresser aux services de l'État; pour un autre, quel qu'il soit, il faut s'adresser au gouvernement et aux services du gouvernement de Nouvelle-Calédonie; pour un autre encore, aux services des Provinces. Les petits États insulaires, qui n'ont pas de représentation à Nouméa ni à Papeete, ne savent souvent pas comment contacter la personne ou le service adéquat quand ils ont besoin de communiquer officiellement.

Il est vrai que la France a l'intention, dans le cadre d'un processus de formation diplomatique, d'envoyer des "délégués " des collectivités au sein des ambassades françaises dans la région (PNG, Fidji, le Vanuatu, l'Australie et la Nouvelle-
Zélande). Dès 2012, il y a eu un délégué pour la Nouvelle-Calédonie au sein de l'ambassade française à Wellington, en Nouvelle-Zélande, et d'autres doivent suivre ailleurs. Notion créatrice et assez innovatrice, sa mise en ouvre est néanmoins délicate, car cette pratique risque de conforter la tendance des petits États insulaires à percevoir les trois collectivités comme de simples instruments de la France. Il faut que celle-ci leur permette de se représenter elles-mêmes, sans un représentant de l'État français à leurs côtés. Les États voisins des collectivités vont juger le comportement de ces délégués, soit comme des représentants des intérêts particuliers des collectivités, soit comme une voix pour la France.

La formation diplomatique d'une manière autonome est même urgente, quand on constate les changements d'intérêts stratégiques dans la région, avec l'accroissement de l'intérêt de la Chine notamment. À noter que celle-ci a maintenant un consulat à Papeete.

Pour mieux équiper les gouvernements locaux, il faut faire plus avec une bonne formation de langue anglaise, et des capacités diplomatiques en rapport avec le style de la région ${ }^{4}$. Un changement récent est à noter, et est le bienvenu : on voit maintenant les délégations des collectivités se déplaçant en Australie et ailleurs, munies de mécanismes de traduction portables.

Il serait utile d'encourager plus d'échanges dans la région, non seulement au niveau plus élevé (comme les visites diplomatiques), mais aussi par le bais de stages assez longs au sein des services publics des pays insulaires de la région, et réciproquement. Inclure les fonctionnaires autochtones serait essentiel, pour mieux souder les liens avec les pays environnants mélanésiens et polynésiens et ainsi ouvrir la voie de l'acceptation régionale.

Assurer un bon et libre échange d'informations entre les collectivités françaises et les États indépendants de la région, dans les deux sens, est fondamental pour renforcer une insertion réussie des collectivités dans leur propre région. Ces dernières années ont vu une augmentation de l'accès à d'excellentes sources d'informations sur les territoires français. La France soutient depuis longtemps le service bilingue de presse Flash d'Océanie/Oceania Flash, pour mieux informer, en français, sur les îles indépendantes et, en anglais, sur les collectivités françaises. Mais le contenu de cet outil très utile a changé au cours des dernières années, en fournissant de moins en

4. «Parler la langue » de la région va au-delà du seul usage de la langue anglaise. Souvent, malheureusement, on continue à entendre l'expression redoutable " anglo-saxon " pour décrire la région du Pacifique Sud (par exemple, le rapport du Sénat de 2013 sur la France dans le Pacifique Sud, utilise ce terme six fois), quand on aurait peut être dû utiliser le mot " anglophone ". Que veut dire " anglo-saxon " quand on parle de l'Australie et de la Nouvelle-Zélande qui sont des pays d'immigrants du monde entier et, pour cette dernière, avec une population océanienne importante ? Quant aux états insulaires de la région, eux-mêmes, tout comme la Nouvelle-Calédonie et la Polynésie française, ils comprennent des peuples composés de nombreuses tribus, avec des centaines de langues et des origines soient mélanésiennes soient polynésiennes. La notion d' "anglo-saxon " n'y a aucune place. Utiliser ce terme ignore la réalité régionale et risque de dégrader ce que la France veut réaliser dans la région. 
moins d'informations sur les développements politiques dans les collectivités françaises, et en représentant de plus en plus un simple sommaire des développements dans les îles indépendantes fourni par les services de presse anglophone. En même temps, et depuis quelques années, les journaux les plus importants des collectivités (Les Nouvelles calédoniennes, La Dépêche de Tahiti) ne sont plus gratuitement accessibles en ligne et les frais d'abonnement sont assez élevés. Ouvrir davantage ces services aux lecteurs, chercheurs et décideurs des États indépendants serait très utile pour augmenter la connaissance et la compréhension mutuelle des collectivités françaises et des pays de la région, surtout en ce moment, où la Nouvelle-Calédonie entame le processus pour définir son futur statut.

\section{Assurer un statut clair et démocratique pour l'avenir}

Même si la France et l'ue clarifient leur système d'aide au développement, et même si la France ouvre le système des tarifs douaniers au commerce régional, et si elle dote les collectivités du soutien administratif pour qu'elles puissent mettre en œuvre leur propre politique dans leurs sphères de compétence, l'acceptation par les voisins régionaux des collectivités françaises dépend finalement d'un principe fondamental : celui de la pleine mise en œuvre, en toute sincérité, par la France qui a le pouvoir souverain, du processus de décolonisation et donc du processus d'autodétermination conforme aux principes de l'onU en Nouvelle-Calédonie et en Polynésie française, les deux collectivités dans lesquelles certains ont exprimé le désir d'accéder à leur propre souveraineté, sous forme d'autonomie et même, pour certains, d'indépendance. Les leaders du forum expriment régulièrement leur soutien à l'autodétermination de la Nouvelle-Calédonie dans leurs communiqués annuels $s^{5}$. Depuis 1998, le forum des Îles du Pacifique s'est donné pour mandat de surveiller le déroulement de l'accord de Nouméa en Nouvelle-Calédonie, et un Comité ministériel y fait des visites régulières (en 1999, 2001, 2004, et 2013).

Dès lors, la meilleure politique pour la France, si elle veut l'acceptation régionale de sa présence, et si elle veut vraiment l'insertion régionale de ses collectivités du Pacifique Sud, serait de remplir, justement et pleinement, ses responsabilités et ses engagements inscrits dans l'accord de
Nouméa. La France, et les parties qui ont signé l'accord, s'approchent de la grande étape finale d'un processus innovateur et unique, et elles progressent bien (voir notre analyse, Fisher, 2012b). Mais nous nous approchons maintenant du dénouement. C'est aux partenaires qui travaillent ensemble en Nouvelle-Calédonie de continuer à mettre en œuvre non seulement la lettre mais aussi l'esprit de cet accord si durement gagné. Nous tous dans la région attendons le processus de référendum si souvent promis, si souvent différé, qui a si souvent échoué dans le passé.

Et en Polynésie française, le moment est assurément venu pour la France d'accepter le rôle des Nations unies. L'État a laissé passer une occasion de démontrer sa bonne foi, lorsque les Polynésiens, menés par Oscar Temaru et soutenus par trois petits États insulaires, ont demandé la réinscription de la Polynésie française auprès du comité de décolonisation de l'onu, une demande satisfaite en 2013 par le vote de l'Assemblée générale de l'onu (A/Rés/67/265, Nations unies, 2013). La mise en ouvre de l'accord de Nouméa a permis à la République de constater qu'elle n'avait rien à redouter de l'engagement de l'ONU dans le processus de décolonisation. Et il est certain qu'avec le soutien des populations de ces collectivités, au moins deux des trois options reconnues par les Nations unies - l'indépendance, l'association ou l'intégration - devraient êtres acceptables pour la France, surtout avec toute son expérience d'innovation statutaire des dernières années. Le fait que la Résolution 67/265 ait été approuvée par tous les membres de l'onu sauf cinq (la France, les États-Unis, le Royaume-Uni, les Pays-Bas, l'Allemagne - dont tous sauf le dernier ont des territoires d'outremer), souligne les aspirations de la communauté internationale. La réaction immodérée d'opposition par la France après le vote à l'onu, que le ministère des Affaires étrangères a caractérisé d' "ingérence flagrante " (ministère des Affaires étrangères, 2013), ne contribue pas à rassurer les petits pays de la région sur la bonne foi de la France.

Celle-ci peut choisir de devenir, elle-même, un partenaire valable de la région, et dans la région. Ou bien elle peut décider de se comporter simplement comme une grande puissance extérieure. Mais ce qui est certain, c'est que ses trois collectivités, elles, sont de la région et dans la région et elles vont y rester, peu importe les choix de la France.

5. Depuis 1998 dans leurs communiqués. Le communiqué du 44e forum, 3-6 septembre 2013, a simplement cité et soutenu le rapport du Comité ministériel du forum après sa visite en Nouvelle-Calédonie les 17-19 juillet 2013. Ce rapport a conclu que "l'esprit et la lettre de l'accord de Nouméa ont été respectés et actés en Nouvelle-Calédonie et que le processus irréversible en vue de l'auto-gouvernance, dans le cadre de l'accord de Nouméa, progresse bien, avec l'engagement de toutes les parties en présence pour un avenir paisible et prospère pour tous les Néo-Calédoniens ». 
Dès lors, c'est à la République et aux populations des collectivités de choisir.

\section{BIBLIOGRAPHIE}

AUSTRALIAN MINISTER FOR FOREIGN AFFAIRS, 2009 (12 May). Press release.

Calvignac Chloé, 2013 (17 janvier). Intervention, colloque La France dans le Pacifique: quelle vision pour le XXI siècle, rapport d'information $n^{\circ} 293$, Sénat session ordinaire 20122013, pp. 55-57 (http://www.senat.fr/rap/ r12-293/r12-2931.pdf).

Conseil constitutionnel, 2004 (12 février). Décision $n^{\circ}$ 2004-490 DC, loi portant statut d'autonomie de la Polynésie française (http://www.conseil-constitutionnel.fr/ conseil-con..ecision-n-2004-490-dc-du-12fevrier-2004.892.html)

Commission du Pacifique Sud (crs), www.SPC.int.

Commission de l'Union européenne, 2006. Commission of the European Communities, Eu Relations with the Pacific Islands a Strategy for a Strengthened Partnership, Communication from the Commission to the Council, Brussels.

—, 2009 (6 novembre). Commission of the European Communities, Elements for a new partnership between the $E U$ and the overseas countries and territories COM 2009, 623 Final Brussels. Dfat, 2015a (13 October). Parliamentary Secretary visit to South Pacific, Press release, Department of Foreign Affairs and Trade.

—, 2015b. Country Brief, Department of Foreign Affairs and Trade (www.dfat.gov.au, consulté le 15 janvier 2015).

Fisher, Denise, 2012a (May). France in the Pacific: Countdown for New Caledonia: review of implementation of the Nouméa accord, Briefing paper $\mathrm{n}^{\circ}$ 2, Centre for European Studies, Australian National University, 3.

-, 2012b (August). France, the eu and the South Pacific, Briefing paper, Centre for European Studies, Australian National University, 3, no 9 .

-, 2013. France in the South Pacific: power and politics, Canberra, ANU Press.

GOUVERNEMENT DE LA NOUVELLE-CALEDONIE, http://www.gouv.nc/.

Hollande François, 2012 (19 novembre). Discours devant les maires d'Outre-mer, Paris (http://www.elysee.fr/declarations/article/ discours-du-president-de-la-republique-devant-les-maires-d-outre-mer/).

INSTITUT NATIONAL DES STATISTIQUES ET DES ÉTUDES ÉCONOMIQUES (INSEE), http://www. insee.fr/fr/.

InSEe Site web. Institut de la Statistique et des Études Économiques.

Kochenov Dimitry (ed.), 2011. The EU Law of the Overseas: Outermost regions, Associated Overseas Countries and Territories, Territories Sui Generis, The Netherlands, Kluwer Law International BV.

LOI ORGANIQUE POLYNÉSIE FRANÇAISE, 2004 (2 février). Loi $n^{\circ}$ 2004-193 (http://www.legifrance.gouv.fr/affichLoiPubliee.do?idDocum ent=JORFDOLE000017759498\&type $=$ gen eral).

LAURIN Elisabeth2013 (17 janvier). Intervention, colloque La France dans le Pacifique: quelle vision pour le XXI siècle, rapport d'information $n^{\circ} 293$, Sénat session ordinaire 20122013, pp. 27-30 (http://www.senat.fr/rap/ r12-293/r12-2931.pdf).

Lurel, Victorin, 2013 (6 juin). Tahiti-pacifiques.

Ministère des Affaires ÉTRANGÈres, 2010. www.diplomatie.gouv.fr (consulté les 19 mai et 8 décembre 2010).

—, 2013 (22 mai). Onu/Polynésie française, réponse du ministre délégué chargé des affaires européennes, M. Thierry Repentin, à une question d'actualité à l'Assemblée nationale, Paris.

Mrgudovic Nathalie, 2010. Les territoires français d'Océanie: une intégration régionale croissante, in Jean-Yves Faberon et Armand Hage (éds), Mondes océaniens. Études en l'honneur de Paul de Deckker, Paris, L'Harmattan, pp. 199-218.

Nations unies, 2013 (17 mai). L'autodétermination de la Polynésie française, résolution A/67/265 adoptée par l'Assemblée générale (http://www.un.org/fr/ga/67/resolutions. shtml).

$\mathrm{Nz}$ AID. www.nzaid.gov.nz consulté le 6 juillet 2010 .

PIf, 2013. Visit of the Pacific Islands Forum Ministerial Committee on New Caledonia, Press Statement 42/13, Pacific Islands Forum, 17-19 July (www.forumsec.org consulté le 14 août 2013).

SARAMANDis Theodore, 2013 (17 janvier). Intervention, colloque La France dans le Paci- 
fique: quelle vision pour le XXI siècle, rapport $d^{\prime}$ information $n^{\circ} 293$, Sénat session ordinaire 2012-2013, pp. 47-51 (http://www.senat.fr/ rap/r12-293/r12-2931.pdf).

SÉnAT (Commission des Affaires étrangères), 1996 (27 mars). Projet de loi autorisant la ratification de la convention portant création du programme régional océanien de l'environnement, rapport n ${ }^{\circ} 256$ 1995-96 (http:// www.senat.fr/leg/pj195-256.html, consulté le 14 aout 2013).
- (Délégation sénatoriale à l'outre-mer), 2013a (17 janvier). La France dans le Pacifique : quelle vision pour le XXI siècle, colloque, rapport d'information $n^{\circ} 293$, Sénat session ordinaire 2012-2013 (http://www.senat.fr/ $\mathrm{rap} / \mathrm{r12}-293 / \mathrm{r} 12-2931 . \mathrm{pdf})$.

- (commission des lois), 2013b (24 juillet). Projet de loi de finances pour 2013: Collectivités d'outre-mer, Nouvelle-Calédonie et TAAF (http://www.senat.fr/rap/a12-154-3/ a12-154-3.html, consulté le 14 août 2013). 